\title{
The trunk muscles behavior in women with low back pain in the test of flexion and extension of the trunk
}

\author{
Guilherme Thomaz de Aquino Nava', Beatriz Mendes Tozim', Mary Hellen Morcelli², Marcelo Tavella Navega ${ }^{2,3}$
}

\begin{abstract}
INTRODUCTION: Chronic low back pain has a direct repercussion on the activities of daily living and the alteration of motor control is its main cause. This change leads to instability of the trunk stabilizer systems that control movements. Trunk flexion and extension movements associated with external disturbances may increase the potential for motor control error, resulting in injury and pain. OBJECTIVE: To evaluate and compare the recruitment of the trunk stabilizer muscles, with and without load, in the flexion and extension movements of the trunk. METHODS: Thirty - four sedentary women were evaluated, aged between 30 and 59 years, divided into: low back pain group (LBPG $n=19)$ and control group (CG $n=15)$. The protocol consisted of clinical evaluation, pain evaluation, strength test of extensor trunk muscles and trunk flexion and extension tests (TFET). Muscle electromyography in internal oblique (IO), lumbar multifidus (LM), rectus abdominis (RA), external oblique (EO) and lumbar iliacus (LI) were performed simultaneously. Multivariate analysis of variance was used with two-way repeated measurements. RESULTS: LBPG presented a trend of greater recruitment in all muscles and loads evaluated, with muscle activation up to $47 \%$ higher when compared to CG. In the intergroup comparison, the global muscles demonstrated a tendency for greater activation in LBPG, with values up to $53 \%$ higher when compared to CG, and predominantly high effect size $\mathrm{d}>0.80$. In the intragroup comparison, the IO, EO, LI and LM muscles of the LBPG presented ascending muscle recruitment with increased load the opposite was seen in the CG, which showed increased activation only in the LI and LM muscles. CONCLUSION: Women with low back pain need more muscle recruitment to remain stable and, in challenging situations, use inefficient strategies.
\end{abstract}

Key words: Lumbar Pain. Postural Balance. Electromyography. Applied Kinesiology.

\section{INTRODUCTION}

Lumbar pain directly affects activities of daily living (ADL) and manifests itself as one of the main musculoskeletal dysfunctions ${ }^{(1-4)}$. When the persistence of low back pain is greater than three months, it is classified as chronic low back pain (CLBP) $)^{(2,4)}$. The main cause of CLBP is muscular dysfunction, which leads to changes in motor control and, as a consequence, inadequate recruitment of the trunk muscles, especially during voluntary activities ${ }^{(1,5-7)}$. Changes in motor control interfere with the ability to detect conditions of instability and to anticipate or respond to these disorders adequately, making subjects susceptible to injury ${ }^{(5,6)}$.

The CLBP is usually reported during active and voluntary lumbar spine movements, since these movements require coordination of the trunk stabilizing muscles, they are divided into global and local muscles according to their functions, in order to generate intervertebral control and postural stability ${ }^{(1,8-11)}$. These disturbance-associated movements, such as the addition of external load, increase the potential error in motor control(12), it causes instability of the systems and may lead to spinal injury and pain ${ }^{(13,14)}$.

The flexion and extension movements of the trunk, from the standing position are extremely recurrent both in the $A D L$, in the work activities as well as in the sports gestures ${ }^{(13)}$. They occur in the sagittal plane and are governed by a complex system ${ }^{(1,14)}$, it must show synergy in the activation of the muscles to promote spinal control and stability while the movements are performed ${ }^{(9)}$. Accordingly, for the investigation of voluntary movements, such as flexion and extension of the trunk, it is necessary to evaluate both the local stabilizing muscles and the global stabilizing muscles to determine the motor behavior ${ }^{(9,14)}$.

The literature describes surface electromyography (EMG) as a tool that has characteristics allowing the function evaluation of the trunk muscles of healthy and injured subjects during voluntary activities ${ }^{(1,15)}$. Therefore, the present study aim to evaluate and compare the recruitment of the trunk

Corresponding Author: Marcelo Tavella Navega. Address: Av. Hygino Muzzi Filho, 737. Mirante. Zipe Code: 17525-000 - Marília - São Paulo Phones: +55 14 3402-1300 / 3402-1310 Email address: gtanava@gmail.com

2 Department of Physical Therapy and Occupational Therapy, Philosophy and Sciences College, Universidade Estadual Paulista (UNESP), Marília (SP), Brazil.

${ }^{3}$ Postgraduate Program in Human Development and Technologies, Institute of Biosciences, Universidade Estadual Paulista (UNESP), Rio Claro (SP), Brazil. Full list of author information is available at the end of the article.

Support source: None.

Submission Date 20 June 2017; Date of acceptance 21 November 2017; Publication Date 28 December 2017 
stabilizing muscles in the flexion and extension movements of the trunk in women with CLBP and to evaluate if the gradual increase of external load influences this recruitment.

\section{METHODS}

This transversal, observational study protocol followed the ethical principles outlined by the Research Ethics Committee of Philosophy and Sciences College of UNESP / Marília (protocol no. 1,054,270). All the participants were informed about the research and signed the Informed Consent Term.

\section{Participants}

34 women aged between 30 and 59 years, of whom 19 comprised the group with CLBP (LBPG, $n=19$ ) according to personal report and pain location. The control group consisted of 15 participants who did not present low back pain (CG, $n=15)$. The eligibility criteria for LBPG were not to practice any physical activity and to present chronic idiopathic low back pain for at least three months prior to the study, for the CG was not to practice any physical activity and did not present a previous report of low back pain. The criteria of non-eligibility for both groups were: nerve compression for lower limbs, pregnancy, history of severe cardiorespiratory problems, lower limb discrepancy, rheumatologic, neurological or vestibular diseases, present surgery in the lumbar spine region, present uncorrected visual alteration or not being able to understand the task. The exclusion criteria were not able to perform some stage of data collection or any intercurrence related to the collection procedure $(n=3)$. The sample was calculated using the $\mathrm{G} *$ Power software, based on the pilot study (data of five participants in each group), using power of 0.95 , probability of error $\alpha$ of 0.05 and size of effect of 1.614, in the comparison of lumbar iliocostal muscle recruitment between LBPG and CG, estimating the need for 12 participants per group.

\section{Procedures}

The data collection procedure consisted of clinical evaluation, dorsal muscle strength test using the dorsal dynamometer, and physical test of flexion and extension of the trunk. The clinical evaluation consisted of data collection on lower limb dominance, it was used to place the electrodes for the EMG evaluation. The dominance was evaluated through the step up and down test, kicking a ball on the target and the anterior displacement test ${ }^{(16)}$. Pain-related information, such as location, time of onset, intensity of pain, presence of paresthesia and use of medication for pain control were also collected. Pain intensity was quantified using the visual analogue scale (VAS). The VAS is formed by a horizontal line of 100 millimeters, with the words "no pain / discomfort" and the words "the worst pain / discomfort imaginable" ${ }^{(4,17)}$ on the right end. Participants were asked to indicate the intensity of pain felt at the time of the evaluation and on a daily basis ${ }^{(17)}$.
At the end, measurement with a ruler occurred to quantify pain intensity ${ }^{(18)}$.

After the clinical evaluation, the muscle strength test (MST) of the extensor muscles of the trunk was performed. The MST was designed to determine the maximum strength of the extensor muscles of each participant, thus standardizing and determining the percentage of load that each one held during the trunk flexion and extension tests (TFET). In the MST, the participants were instructed to place their feet in the place determined by the dorsal dynamometer and to perform traction force in the direction of trunk extension, not to force with the upper limbs and not flex the lower limbs ${ }^{(3)}$. The MST is best seen in figure 1 .

The test was performed in two different moments for collection, for familiarization, and the highest value obtained was used for analysis ${ }^{(19)}$. Participants were verbally stimulated and maintained contraction for 4 seconds, with 1 minute rest ${ }^{(15.3)}$. Hence, the TFET was perfomed, which initially consisted of neutral foot positioning, bare feet, hip width, relaxed and stretched arms and hands together ${ }^{(12)}$. After the

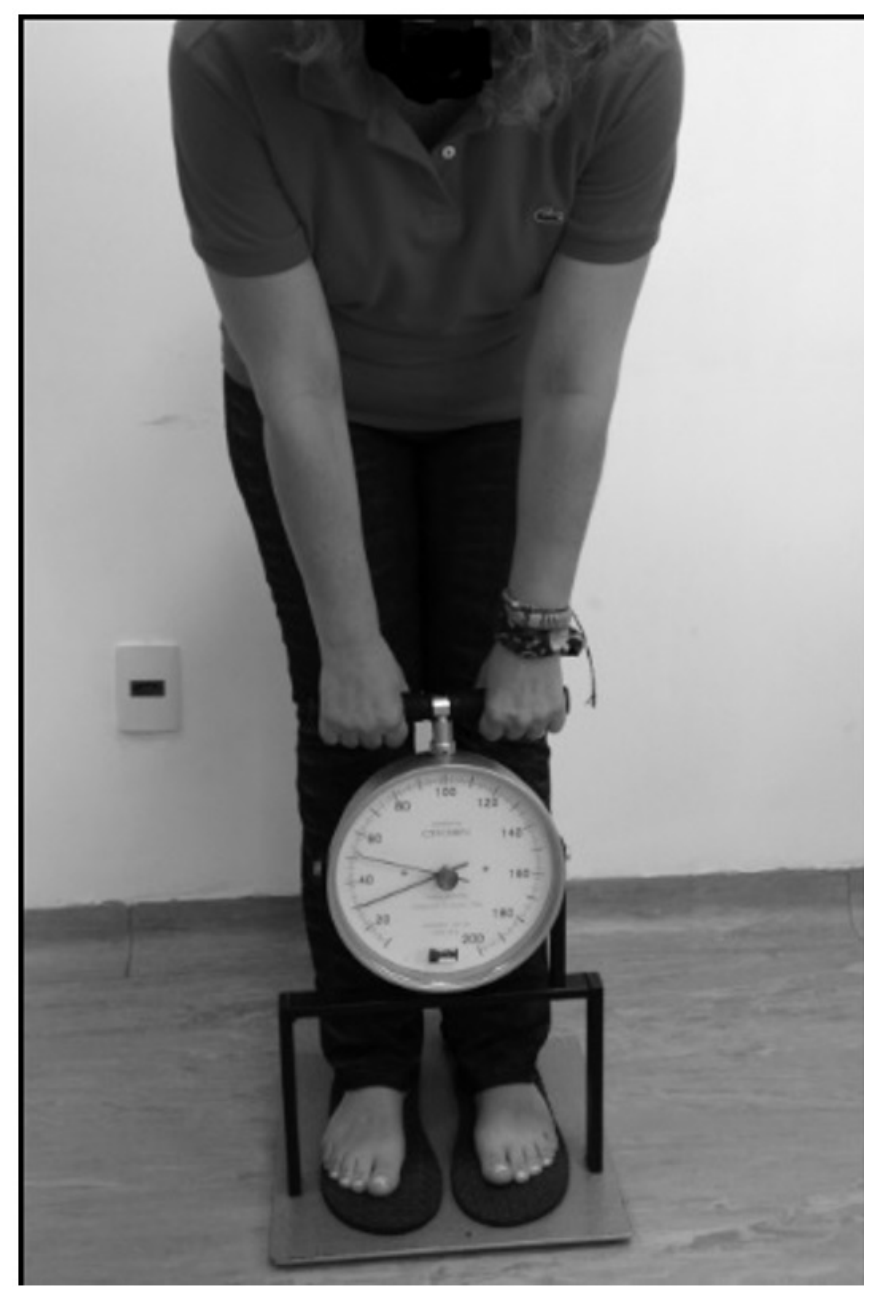

Figure 1. Muscle strength test 
positioning they performed the maximum anterior flexion of the trunk, keeping the arms loose and the head following the movement ${ }^{(12)}$. They were oriented to perform the flexion with the same amplitude, thus maintaining the reproducibility and reliability of the same distance in all the tests ${ }^{(7,12,13)}$.

The TFET consisted of three cycles, each cycle comprising a flexion and extension, totaling three flexion movements and three trunk extension movements, with two minutes of rest between cycles ${ }^{(7,12)}$. The collection of the EMG signal, concomitant to the TFET, lasted 42 seconds running for each participant. In the first six seconds, the participants remained standing still. Subsequently, they performed three cycles of flexion and extension of the trunk, divided by the verbal stimuli of "incline" and "return". Each cycle had a duration of 12 seconds, six seconds for trunk flexion and six seconds for trunk extension, as can be seen in Figure $2^{(7,13)}$.

In the first phase of TFET, the participants performed the three cycles without adding additional load. In the second phase, they performed the three cycles holding a load quantity equivalent to $5 \%$ of the maximum capacity obtained in the MST and, in the last phase, carried out three cycles of movement holding a load equivalent to $10 \%$ of the maximum capacity reached in the MST. The load increase was done through free weights ranging from 50 grams to $2 \mathrm{~kg}$ and added according to the MST. The loads were placed in a bag and wrapped in such a way they did not move and the participants held bilaterally and with their hands and arms stretched during the TFET ${ }^{(12)}$.

The muscles of each subject were found and the electrodes were positioned unilaterally on the dominant side of the trunk and oriented longitudinally with the fibers of the muscles evaluated $^{(18,20)}$. A tricotomy was performed in the electrode placement areas and gauze skin abrasion was performed to decrease the impedance ${ }^{(1.18)}$. Alcohol was used to clean the region where the electrodes were positioned ${ }^{(21)}$. The surface electrodes used were those of $\mathrm{Ag} / \mathrm{AgCl}$, bipolar, active with pre-amplification of 20 times EMGSystem ${ }^{\circledR}$ (EMG system of Brazil, São José dos Campos, SP, Brazil). They were positioned in bipolar configuration, capture area of $1 \mathrm{~cm}$ in diameter and distance of $2 \mathrm{~cm}$ between each electrode ${ }^{(21)}$. A reference electrode was positioned on the styloid process of the ulna ${ }^{(1.22)}$, with the aim of capturing the electromyographic signals, the acquisition of biological signals model EMG830c EMGSystem ${ }^{\circledR}$ (EMG system of Brazil, São José dos Campos (SP), Brazil) of 8 channels, software for collecting EMGLab, data processing and storage, calibrated with sampling frequency of $2000 \mathrm{~Hz}$, total gain of 2000 times (20 times in the sensor and 100 times in the equipment) and system impedance $10^{\circ} \Omega$, common rejection module $>100 \mathrm{~dB}$ and signal noise ratio $<3 \mu \mathrm{V}$ RMS. The participants were placed in dorsal decubitus for the location and marking of the muscles:

- Rectus abdominis muscle (RA): $1 / 2 \mathrm{~cm}$ of the distance between the xiphoid process and the umbilical scar, approximately $3 \mathrm{~cm}$ lateral to the midline ${ }^{(18)}$.

- Internal oblique muscle (IO): 2 centimeters medial and inferior to the anterior superior iliac spine (ASIS) (21).

- External oblique muscle (EO): $50 \%$ of the distance between the lower rib cage and $\operatorname{ASIS}^{(21)}$.

Afterwards, they were placed in a ventral decubitus position on a wooden box, made to perform the maximum voluntary isometric contraction (MVIC) and to locate and mark the muscles:

- Lumbar iliocostal muscle (LI): 6 centimeters lateral to the space between the spinal processes of $L 2-L 3^{(21)}$.

- Lumbar multifidus muscle (LM): Placed on the line connecting the posterior superior iliac spine and the space between $L 1-L 2$ at the level of $L 5^{(18)}$.

Besides the previously mentioned tests, the MVIC was collected. The RA muscle was evaluated with the participants sitting on the box, with flexed knees at 90ㅇ, feet supported and lower limbs (LL) attached ${ }^{(15)}$. The upper limbs (UL) were crossed in front of the chest and the trunk slightly inclined backwards ${ }^{(15)}$. Feet and trunk were stabilized by the evaluators and participants performed maximum strength to flex the trunk $^{(15,18)}$.

The IO and EO muscles were evaluated in lateral decubitus with the side to be evaluated upwards. The knees were positioned flexed at 90, the UL joined and crossed in front of the trunk ${ }^{(15)}$. The evaluators stabilized the knees and hips,

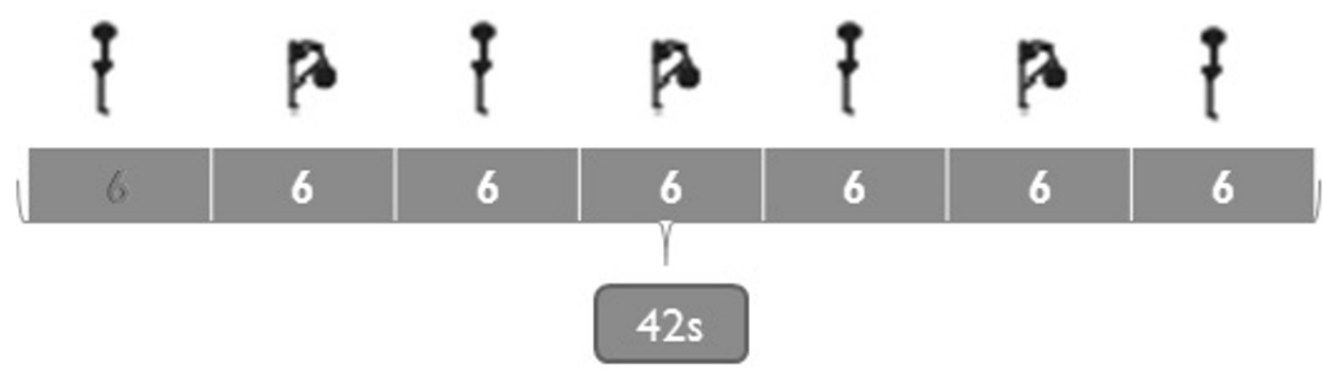

Figure 2. Protocol adopted in the Test of flexion and extension of the trunk. 
shoulders and elbows, and the participants strengthened lateral bending of the trunk ${ }^{(15,18)}$. For the purpose of evaluating the $\mathrm{LI}$ and LM muscles, the participants were positioned in the ventral decubitus, with the LL kept on the box, from the ASIS region to the feet, and the trunk remained outside the box ${ }^{(15,23)}$. The participants were stabilized in the ankle, popliteal fossa and hip region by velcro straps ${ }^{(23)}$. Another velcro strap was placed in the region of the shoulder blades to limit the extension of the trunk and the participants were verbally stimulated to maximize force in the direction of trunk extension ${ }^{(15,18)}$. Each MVIC was performed twice with isometric muscle contraction and for four seconds, with one minute rest ${ }^{(24)}$. The highest value was used to analyze the data ${ }^{(24)}$. The verbal stimulus was given throughout the MVIC, in addition to guidance on which muscle would be evaluated for better recruitment.

\section{Analysis of electromyographic data}

The EMG analysis was performed using the software Matlab ${ }^{\circledR}$ version 7.1 and analyzed during the three cycles of flexion and extension of the trunk with and without load, i.e, it was used separately, linear envelope for the $0 \%$ load, for the load 5\% and for the load with 10\%. Four-order Butterworth high pass filter with $20 \mathrm{~Hz}$ cut-off frequency, 4 th order Butterworth low pass filter with $500 \mathrm{~Hz}$ cut-off frequency, full wave signal rectification and low pass filter with $6 \mathrm{~Hz}$ cut-off frequency were used to form the linear envelope. The linear envelope values of the muscles were normalized by the highest value obtained in the MVIC of their respective muscle.

\section{Statistical analysis}

Statistical analysis was performed using PASW 18.0 (SPSS Inc., Chicago, USA). Data were presented as mean and standard deviation. The Shapiro-Wilk test was used to verify normality and multivariate analysis of variance (MANOVA) to compare the characteristics of the participants. Considering that the age and body mass index (BMI) of the subjects presented significant difference between the groups, both were used as co-variables for the statistical analysis of the dependent variables, loads added. For the analysis of the variables the co-variance analysis was used with two-way repeated measures (ANCOVA), considering the groups as independent factor and the loads as repeated measurements. The ANOVA was followed by the post-hoc Bonferroni. The significant value adopted was $p<0.05$. The effect size (Cohen's d) was calculated for the dependent and independent variables in LBPG and CG, which is found in equation 1. M1 represents the mean of the LBPG, M2 is the CG average and the DP is the combined standard deviation ${ }^{(2.25)}$. The classification used was: low effect 0.20 , medium 0.50 and value equal to or higher than 0.80 as a high effect, analyzed using the $\mathrm{G} *$ Power software ${ }^{(2,25,26)}$.

$$
d=\frac{M_{1-} M_{2}}{D P}
$$

\section{RESULTS}

Table 1 presents the results of the characteristics of LBPG and CG participants. The mean age of CG was 7 years lower compared to LBPG, in addition to presenting lower BMI. For this reason, age and $\mathrm{BMI}$ were used as co-variables in the statistical analyzes for the comparison of groups. Table 2 presents the muscular activation of LBPG and CG in different loads $(0 \%, 5 \%$ and $10 \%)$. No significant differences were observed in the interaction (Group X Load). Despite the absence of statistically significant differences in the interaction, the effect size analysis showed important data in the inter-and intra-group comparison. Cohen's d values in the intergroup comparison for each load (0\%, 5\% and $10 \%)$ are presented in Table 3 and in Table 4 are the Cohen's d values in the intragroup comparison (comparing the $0 \%$ load with $5 \%$, comparing the load $0 \%$ with $10 \%$, and comparing the load $5 \%$ with $10 \%$ ) for both groups.

Table 1. Demographic characteristics, pain and strength test of trunk extensor muscles

\begin{tabular}{|c|c|c|c|c|}
\hline & LBPG & CG & MANOVA & MANOVA \\
\hline & $(n=19)$ & $(n=15)$ & value of $p$ & value of $F$ \\
\hline Age (years) & $46.31 \pm 8.47$ & $39.8 \pm 8.01$ & 0.029 & $5,194 *$ \\
\hline $\mathrm{BMI}\left(\mathrm{Kg} / \mathrm{m}^{2}\right)$ & $27.72 \pm 4.69$ & $24.18 \pm 3.44$ & 0.020 & $5,973^{*}$ \\
\hline $\begin{array}{l}\text { Duration of low } \\
\text { back pain (years) }\end{array}$ & $8.84 \pm 9$ & - & - & \\
\hline daily pain (VAS) & $3.97 \pm 2.61$ & - & - & \\
\hline $\begin{array}{l}\text { Pain in assessment } \\
\text { (VAS) }\end{array}$ & $0.89 \pm 1.67$ & - & - & \\
\hline $\begin{array}{l}\text { Strength of trunk } \\
\text { extensor muscle } \\
\text { (kgf) }\end{array}$ & $62.05 \pm 11.6$ & $58.06 \pm 11.39$ & 0.326 & 0.997 \\
\hline
\end{tabular}

Note: LBPG: low back pain group; CG: control group; KG: kilograms; BMI: Body mass index; M: meters; KGF: kilogram-force. * significant difference $(p<0.05)$.

Table 2. Linear data of normalized muscle activation in the TFET, with and without load.

\begin{tabular}{|c|c|c|c|c|c|c|}
\hline & \multicolumn{3}{|c|}{ LBPG } & \multicolumn{3}{|c|}{ CG } \\
\hline & $0 \%$ & $5 \%$ & $10 \%$ & $0 \%$ & $5 \%$ & $10 \%$ \\
\hline 10 & $0.36 \pm 0.11$ & $0.41 \pm 0.16$ & $0.38 \pm 0.14$ & $0.31 \pm 0.12$ & $0.30 \pm 0.13$ & $0.28 \pm 0.12$ \\
\hline EO & $0.67 \pm 0.63$ & $0.59 \pm 0.48$ & $0.79 \pm 0.74$ & $0.37 \pm 0.27$ & $0.35 \pm 0.23$ & $0.37 \pm 0.31$ \\
\hline LI & $0.17 \pm 0.07$ & $0.21 \pm 0.07$ & $0.26 \pm 0.07$ & $0.09 \pm 0.02$ & $0.14 \pm 0.06$ & $0.18 \pm 0.07$ \\
\hline RA & $0.26 \pm 0.15$ & $0.27 \pm 0.15$ & $0.26 \pm 0.17$ & $0.18 \pm 0.05$ & $0.17 \pm 0.07$ & $0.16 \pm 0.05$ \\
\hline LM & $0.23 \pm 0.07$ & $0.30 \pm 0.10$ & $0.33 \pm 0.09$ & $0.19 \pm 0.06$ & $0.24 \pm 0.08$ & $0.27 \pm 0.08$ \\
\hline
\end{tabular}

Note: LBPG: low back pain group; CG: control group; IO: internal oblique muscle; EO: external oblique muscle; LI: lumbar iliocostal muscle; RA: rectus abdominis muscle and LM: lumbar multifidus muscle. $0 \%$ : No extra charge; $5 \%$ : $5 \%$ of the maximum load reached in the MST; $10 \%: 10 \%$ of the maximum load reached in the MST. 
Table 3. Cohen's d values in the comparison between LBPG and CG in the same loads.

\begin{tabular}{cccc}
\hline & $\mathbf{0 \%}$ & $\mathbf{5 \%}$ & $\mathbf{1 0 \%}$ \\
\hline IO & 0.43 & 0.75 & 0.76 \\
EO & 0.61 & 0.63 & 0.74 \\
LI & 1.55 & 1.07 & 1.14 \\
RA & 0.49 & 0.85 & 0.79 \\
LM & 0.61 & 0.66 & 0.7 \\
\hline
\end{tabular}

Note: IO: internal oblique muscle; EO: external oblique muscle; LI: lumbar iliocostal muscle; RA: rectus abdominis muscle and LM: lumbar multifidus muscle. 0\%: No extra charge; $5 \%$ : $5 \%$ of the maximum load reached in the MST; $10 \%: 10 \%$ of the maximum load reached in the MST.

Table 4. Cohen's d values in intragroup comparisons and between different loads.

\begin{tabular}{ccccccccc}
\hline & \multicolumn{3}{c}{ LBPG } & & \multicolumn{3}{c}{ CG } \\
\cline { 2 - 3 } \cline { 7 - 8 } \cline { 7 - 8 } & $\mathbf{0 - 5} \%$ & $\mathbf{0 - 1 0 \%}$ & $\mathbf{5 - 1 0 \%}$ & & $\mathbf{0 - 5} \%$ & $\mathbf{0 - 1 0 \%}$ & $\mathbf{5 - 1 0 \%}$ \\
\hline IO & 0.36 & 0.15 & 0.19 & & 0.07 & 0.25 & 0.15 \\
EO & 0.14 & 0.17 & 0.32 & & 0.07 & 0.00 & 0.07 \\
LI & 0.57 & 1.28 & 0.71 & & 1.11 & 1.74 & 0.61 \\
RA & 0.06 & 0.00 & 0.00 & & 0.16 & 0.40 & 0.16 \\
LM & 0.81 & 1.24 & 0.31 & & 0.7 & 1.13 & 0.37 \\
\hline
\end{tabular}

Note: LBPG: low back pain group; CG: control group; IO: internal oblique muscle; EO: external oblique muscle; LI: lumbar iliocostal muscle; RA: rectus abdominis muscle and LM: lumbar multifidus muscle. $0 \%$ : No extra charge; $5 \%$ : $5 \%$ of the maximum load reached in the MST; $10 \%: 10 \%$ of the maximum load reached in the MST.

\section{DISCUSSION}

The aim of the present study was to verify if the IO, EO, RA, $\mathrm{LI}$ and $\mathrm{LM}$ muscles presented differences in muscle recruitment in women with idiopathic CLBP in the TFET and, secondarily, to assess whether the gradual increase in external load influenced in this recruitment. It was found that LBPG tends to present higher levels of muscle activation in all loads and in all evaluated muscles, although no statistically significant results were observed $(p<0.05)$. A possible reason for the data is not significant is that the LBPG presented low intensity of pain, with VAS of $0.89 \pm 1.67 \mathrm{~cm}$ on the day of the test. When we observed the electromyographic percentage values we noticed greater muscle activation in the LBPG when compared to the $\mathrm{CG}$, for this reason, the effect size analysis was done, although the $\mathrm{p}$ value gives the probability of obtaining a significant statistic, it does not report on the clinical or practical importance of the results ${ }^{(26)}$.

LBPG presented greater muscle recruitment in all loads, when compared to CG, in the TFET. In unloaded TFET, LBPG had $13 \%$ to $47 \%$ more recruitment than CG. When analyzing the different loads in the intergroup comparison, we observed that the EO muscle presented $44 \%, 40 \%$ and $53 \%$ greater muscle activation in the LBPG when compared to the CG in the respective loads $0 \%, 5 \%$ and $10 \%$, while the LI muscle presented respectively $47 \%, 33 \%$ and $30 \%$ and the RA muscle
$30 \%, 37 \%$ and $38 \%$. This same situation could be seen in the size of the effect that yielded predominantly high values $d>0.80$. Clinical practice indicates the greater recruitment of the muscles that make up the overall system seems to be associated with an increase in the load on the spine ${ }^{(7,9,27)}$, which presents a potentially harmful and worsening pain level ${ }^{(1,8)}$. Increase in muscle recruitment values do not necessarily indicate greater stability of the spine ${ }^{(28)}$, since this strategy known as trunk rigidity strategy aims to increase the safety and protection of the spine, without positive results ${ }^{(8,9,28)}$. This increased muscle rigidity in LBPG can also be explained by muscle strength deficits of this same group, since if LBPG and CG needed the same muscular strength to maintain stability during the TFET, it is probable the LBPG required more recruitment to produce the necessary force ${ }^{(6)}$. However, this is a limitation of the study, since muscle strength has not been evaluated.

In the intragroup comparison, values of muscle activation and effect size were higher as the external load increased in the LBPG in all muscles evaluated, a situation that was not observed in the CG. These findings may be due to a decrease in passive stability and changes in the proprioceptive information of lumbar tissues, which are evidenced by an increase in demand ${ }^{(6)}$ with external loads, making TFET more challenging and revealing motor control deficits ${ }^{(1,7,10-12,29)}$. The CG presented increased recruitment of only the LI and LM muscles. This result is expected since the increase in anterior load increases the requirement of the extensor muscles of the trunk ${ }^{(7,29,24)}$. The absence of alterations in the percentage values of IO, EO and RA muscles, according to the increase of load in the CG, indicates little modification in the pattern of muscle activation and better efficiency in the redistribution of muscle demand in this group, with a lower probability of injury or pain, even in more challenging situations ${ }^{(1,27)}$.

In the LI and LM muscles there were the same recruitment trend in both groups, however, percentage values and effect size showed greater activation in the LBPG. This increase may be related to the theory of pain adaptation ${ }^{(30)}$, where there is facilitation of the motor neuron inhibitory pathway of the IO, EO and RA muscles and excitation of the motoneuron pathway of the $L I$ and $L M$ muscles, and with this increase in recruitment of these muscles, in order to reduce the amplitude and speed of movement with pain ${ }^{(12,18,22,24,30)}$.

\section{CONCLUSION}

Women who present chronic idiopathic low back pain need greater muscle activation to maintain spine stability and, with increased load, have used inefficient neuromuscular strategies to remain stable. The findings of this study guide clinical practice, demonstrating the importance of muscle training, centred on the local muscles, and it should be associated with neuromuscular reeducation of the movements, which may result in the reduction of spinal loads. Notwithstanding, 
studies with a practical focus should be performed to verify such hypothesis.

\section{AUTHORS CONTRIBUTION}

GTAN: Idealizer of the work. BMT: Analyzed and reviewed the data. MHM: Statistical analysis and review. MTN: Advisor and reviewer.

\section{CONFLICT OF INTEREST}

None.

\section{AUTHOR DETAILS}

${ }^{1}$ Institute of Biosciences, Universidade Estadual Paulista (UNESP), Rio Claro (SP), Brazil.

\section{REFERENCES}

1. Rossi DM, Morcelli MH, Marques NR, Hallal CZ, Gonçalves M, LaRoche $\mathrm{DP}$, et al. Antagonist coactivation of trunk stabilizer muscles during Pilates exercises. J Bodyw Mov Ther [Internet]. 2014;18(1):34-41.

2. Mostagi FQRC, Dias JM, Pereira LM, Obara K, Mazuquin BF, Silva MF, et al. Pilates versus general exercise effectiveness on pain and functionality in non-specific chronic low back pain subjects. J Bodyw Mov Ther. 2015;19(4):636-45.

3. Cho KH, Beom JW, Lee TS, Lim JH, Lee TH, Yuk JH. Trunk muscles strength as a risk factor for nonspecific low back pain: A pilot study. Ann Rehabil Med. 2014;38(2):234-40.

4. Luiz A, Teixeira S, Fernandes N, Meneses E. Evaluation of functional disability and pain in patients with chronic low back pain submitted to physiotherapy. MTP\&RehabJournal. 2016;14.

5. Tsao H, Galea MP, Hodges PW. Driving plasticity in the motor cortex in recurrent low back pain. Eur J Pain. 2010;14(8):832-9.

6. Butler HL, Hubley-Kozey CL, Kozey JW. Changes in electromyographic activity of trunk muscles within the sub-acute phase for individuals deemed recovered from a low back injury. J Electromyogr Kinesiol. 2013;23:369-77.

7. Sánchez-Zuriaga D, López-Pascual J, Garrido-Jaén D, García-Mas MA. A Comparison of Lumbopelvic Motion Patterns and Erector Spinae Behavior Between Asymptomatic Subjects and Patients With Recurrent Low Back Pain During Pain-Free Periods. J Manipulative Physiol Ther. 2015;38(2):130-7.

8. Hodges PW, Moseley GL, Gabrielsson A, Gandevia SC. Experimental muscle pain changes feedforward postural responses of the trunk muscles. Exp brain Res. 2003;151(2):262-71.

9. D'hooge R, Hodges P, Tsao H, Hall L, MacDonald D, Danneels L. Altered trunk muscle coordination during rapid trunk flexion in people in remission of recurrent low back pain. J Electromyogr Kinesiol. 2013;23(1):173-81.

10. Panjabi M. The Stabilizing System of the Spine Part I. Journal of Spinal Disorders. 1992;5:383-9.

11. Bergmark A. Stability of the lumbar spine: a study in mechanical engineering. Acta Orthop. 1989;230:1-54.

12. Larivière C, Gagnon D, Loisel P. The effect of load on the coordination of the trunk for subjects with and without chronic low back pain during flexionextension and lateral bending tasks. Clin Biomech. 2000;15(6):407-16.
13. Sánchez-Zuriaga D, Artacho-Pérez C, Biviá-Roig G. Lumbopelvic flexibility modulates neuromuscular responses during trunk flexion-extension. J Electromyogr Kinesiol 2016;28:152-7.

14. Kienbacher T, Paul B, Habenicht R, Starek C, Wolf M, Kollmitzer J, et al. Age and gender related neuromuscular changes in trunk flexion-extension. J Neuroeng Rehabil. 2015;12(1):1-10.

15. Vera-Garcia FJ, Moreside JM, McGill SM. MVC techniques to normalize trunk muscle EMG in healthy women. J Electromyogr Kinesiol. 2010;20(1):10-6.

16. Hoffman M, Schrader J, Applegate T, Koceja D. Unilateral Postural Control of the Functionally Dominant and Nondominant Extremities of Healthy Subjects. 1998;33(4):319-22.

17. Morcelli MH, Faganello FR, Navega MT. Avaliação da flexibilidade e dor de idosos físicamente ativos e sedentários. MTP\&RehabJournal. 2010;8:298-304.

18. Schinkel-Ivy A, Nairn BC, Drake JDM. Investigation of trunk muscle cocontraction and its association with low back pain development during prolonged sitting. J Electromyogr Kinesiol. 2013;23(4):778-86.

19. Gruther W, Wick F, Paul B, Leitner C, Posch M, Matzner M, et al. Diagnostic accuracy and reliability of muscle strength and endurance measurements in patients with chronic low back pain. J Rehabil Med. 2009;41(8):613-9.

20. Marshall P, Murphy B. The validity and reliability of surface EMG to assess the neuromuscular response of the abdominal muscles to rapid limb movement. J Electromyogr Kinesiol. 2003;13(5):477-89.

21. Marques NR, Hallal CZ, Gonçalves M. Padrão de co-ativação dos músculos do tronco durante exercícios com haste oscilatória. Motriz Rev Educ Fis. 2012;18(2):245-52.

22. Graham RB, Oikawa LY, Ross GB. Comparing the local dynamic stability of trunk movements between varsity athletes with and without non-specific low back pain. J Biomech. 2014;47(6):1459-64.

23. Luoto $\mathrm{S}$, Heliijvaara $\mathrm{M}$, Hurri $\mathrm{H}$, Alaranta $\mathrm{H}$. Static back endurance low-back pain and the risk of. 1995;10(6):323-4.

24. Nelson-Wong E, Alex B, Csepe D, Lancaster D, Callaghan JP. Altered muscle recruitment during extension from trunk flexion in low back pain developers. Clin Biomech. 2012;27(10):994-8.

25. Beck $T$ ravis $W$. The importance of a priori sample size estimation in strength and conditioning research. 2013;27(8):2323-37.

26. Espírito-Santo H, Daniel F. Calcular e apresentar tamanhos do efeito em trabalhos científicos: As limitações do $p<0,05$ na análise de diferenças de médias de dois grupos. Rev Port Investig Comport e Soc. 2015;1(1):3-16.

27. Lee PJ, Granata KP, Moorhouse KM. Active trunk stiffness during voluntary isometric flexion and extension exertions. Hum Factors. 2007;49(1):100-9.

28. IAF Stokes, MG Gardner-Morse SH. Abdominal muscle activation increase lumbar spinal stability: analysis of contributions of different muscle groups. Clin Biomech. 2012;26(8):797-803.

29. Dubois J-D, Piché $M$, Cantin V, Descarreaux M. Effect of experimental low back pain on neuromuscular control of the trunk in healthy volunteers and patients with chronic low back pain. J Electromyogr Kinesiol. 2011;21(5):774-81.

30. Lund JP, Donga R, Widmer CG, Stohler CS. The pain-adaptation model: a discussion of the relationship between chronic musculoskeletal pain and motor activity. Can J Physiol Pharmacol. 1991;69(5):683-94. 